Original paper

\title{
Dosimetric effect by shallow air cavities in high energy electron beams
}

\author{
M. Zarza-Moreno ${ }^{\mathrm{a}, \mathrm{b}, *}$, P. Carreira ${ }^{\mathrm{c}}$, L. Madureira ${ }^{\mathrm{c}}$, H. Miras del Rio ${ }^{\mathrm{e}}$, F.J. Salguero ${ }^{\mathrm{d}}$, \\ A. Leal ${ }^{\mathrm{e}}$, N. Teixeira ${ }^{\mathrm{b}}$, A.P. Jesus ${ }^{\mathrm{a}}$, G. Mora ${ }^{\mathrm{a}}$ \\ ${ }^{a}$ Centro de Física Nuclear, Universidade de Lisboa, Lisbon, Portugal \\ ${ }^{\mathrm{b}}$ Escola Superior de Tecnologia da Saúde de Lisboa, Lisbon, Portugal \\ ${ }^{\mathrm{c}}$ Hospital de Santa Maria - Medical Consult, Lisbon, Portugal \\ ${ }^{\mathrm{d}}$ Het Nederlands Kanker Instituut - Antoni van Leeuwenhoek, Amsterdam, Netherlands \\ e Departamento de Fisiología Médica y Biofísica, Universidad de Sevilla, Sevilla, Spain
}

\section{A R T I C L E I N F O}

\section{Article history:}

Received 11 November 2012

Received in revised form

12 July 2013

Accepted 15 July 2013

Available online $\mathrm{xxx}$

\section{Keywords:}

Monte Carlo algorithms

Air cavity

Electron beam

EBT2 Gafchromic film

Pencil beam algorithms

\begin{abstract}
A B S T R A C T
This study evaluates the dosimetric impact caused by an air cavity located at $2 \mathrm{~mm}$ depth from the top surface in a PMMA phantom irradiated by electron beams produced by a Siemens Primus linear accelerator. A systematic evaluation of the effect related to the cavity area and thickness as well as to the electron beam energy was performed by using Monte Carlo simulations (EGSnrc code), Pencil Beam algorithm and Gafchromic EBT2 films. A home-PMMA phantom with the same geometry as the simulated one was specifically constructed for the measurements. Our results indicate that the presence of the cavity causes an increase (up to 70\%) of the dose maximum value as well as a shift forward of the position of the depth-dose curve, compared to the homogeneous one. Pronounced dose discontinuities in the regions close to the lateral cavity edges are observed. The shape and magnitude of these discontinuities change with the dimension of the cavity. It is also found that the cavity effect is more pronounced (6\%) for the $12 \mathrm{MeV}$ electron beam and the presence of cavities with large thickness and small area introduces more significant variations (up to $70 \%$ ) on the depth-dose curves.

Overall, the Gafchromic EBT2 film measurements were found in agreement within $3 \%$ with Monte Carlo calculations and predict well the fine details of the dosimetric change near the cavity interface. The Pencil Beam calculations underestimate the dose up to 40\% compared to Monte Carlo simulations; in particular for the largest cavity thickness $(2.8 \mathrm{~cm})$.
\end{abstract}

(c) 2013 Associazione Italiana di Fisica Medica. Published by Elsevier Ltd. All rights reserved.

\section{Introduction}

The presence of air cavities in regions irradiated with radiotherapy beams is very common in clinical applications, especially in the head and neck region [1,2]. Over the last years, the evaluation of the impact of air inhomogeneities on the absorbed dose has been a subject of many research investigations either by experimental measurements or by using Monte Carlo (MC) simulations [3-12]. All previous works reported that the perturbation caused by the air inhomogeneity near the tissue-air interfaces can be responsible for significant overdosing and underdosing, whose magnitude can be of clinical interest in some cases. Accurate knowledge of this effect becomes critical for treatment planning dose calculations in order to reduce the dose to healthy tissue and organs at risk and to avoid

* Corresponding author. Centro de Física Nuclear, Universidade de Lisboa, Av. Prof. Gama Pinto 2, 1649-003 Lisbon, Portugal. Tel.: +351 217904812; fax: +351 217954288.

E-mail addresses: miriam@cii.fc.ul.pt, miriamzamo@gmail.com (M. Zarza-Moreno). posterior complications in these structures as well as to avoid underdosing of target volumes.

In the early 70s, a systematic study was performed to assess the air effect with the variation in cavity dimension and energy beam by using experimental measurements [9]. The work was performed for electron beams produced by a Siemens Betatron with energies of 10 and $42 \mathrm{MeV}$ and it reported a significant increase of dose (up to $60 \%$ ) adjacent to the air cavity $(1 \mathrm{~cm}$ radius and $2 \mathrm{~cm}$ height located at $1 \mathrm{~cm}$ depth from the surface).

Experimentally the evaluation of the dose perturbation in the presence of air is a complex task due to the difficulty of measuring accurately the air-tissue interface effect. In contrast, Monte Carlo simulations are well suited for these cases as they provide a better assessment of interface doses and they can handle electron multiple scattering in the presence of inhomogeneities much more accurately than any existing analytical dose models, which can give errors in calculated dose as high as $20 \%$ [13].

Accurate dose calculation of the perturbation caused by the presence of air inhomogeneities can be an important factor for the 
widespread clinical used of new electron therapy techniques, such as electron and mixed electron-photon beams intensity modulated therapy, which are expected to improve the conformality of the delivered dose distribution to the target volume.

To date, a large number of works has been published on the influence of air cavities as function of beam energy or cavity configuration for radiotherapy photon beams [3-8]. However, there is a lack of studies accounting the dependence of the air cavity effect on its geometry, position and energy for electron beams [10$12]$. Most of the published studies for electron beams $[14,15]$ were focused benchmarking the accuracy of a commercial treatment planning system incorporating Monte Carlo dose calculation. Although the results of these works have shown a dosimetric effect, both experimentally and with Monte Carlo simulations, in air cavity phantoms for different energy of electron beams, the magnitude of the dose perturbation depending on the geometry and location of the cavity was not reported.

Recently, Chow and Grigorov [12] published an investigation of the dosimetric impact of an air cavity for electron beams by using Monte Carlo calculations. They evaluated the variation of the cavity effect versus beam energy as well as size and position of the cavity, considering always the cavities located at depths deeper than $5 \mathrm{~mm}$. This work also included a comparison of the MC dose distributions with experimental measurements; however, the comparison was restricted for one specific geometry of the inhomogeneous phantom studied in the work.

To our knowledge, the effect of air inhomogeneities shallower than $5 \mathrm{~mm}$ has not been systematically studied. However, shallow cavities can affect the clinical result in several radiotherapy treatments $[14,16]$. Furthermore, Ding et al. [13] reported that the accuracy of pencil beam calculations may depend not only on the geometry of the 3D inhomogeneity, but also on the location of the inhomogeneity.

The goal of present work is to perform a systematic study assessing the air cavity perturbation on electron dose distributions for air cavities located at $2 \mathrm{~mm}$ depth from the phantom surface. The dependence of the effect on cavity size (area and thickness) and beam energy is evaluated by using Monte Carlo simulations (EGSnrc code), pencil beam (PB) algorithm and experimental measurements (Gafchromic EBT2 films) for a $10 \times 10 \mathrm{~cm}^{2}$ field size.

\section{Material and methods}

Phantom geometry

An air cavity phantom of PMMA was specifically built for this investigation. The phantom with an area of $15 \times 15 \mathrm{~cm}^{2}$ and a total height of $12 \mathrm{~cm}$ consisted of two parts: a) a PMMA block $\left(15 \times 15 \mathrm{~cm}^{2}\right.$ area and $2.8 \mathrm{~cm}$ thickness) containing an air cavity of $3.8 \times 3.8 \times 2.8 \mathrm{~cm}^{3}$ at the center and b) 30 PMMA slabs $\left(15 \times 15 \mathrm{~cm}^{2}\right.$ area) with thickness of $0.3 \mathrm{~cm}$ placed below previous part (a). Inside the $3.8 \times 3.8 \times 2.8 \mathrm{~cm}^{3}$ cavity, air cavities with varying areas $S$ and thicknesses $L$ (Fig. 1a and b) were arranged using small solid blocks of PMMA. The dimensions of these cavities were chosen as representative geometries of cavities presented in the head and neck region $[7,14]$ and are summarized in Table 1 . For all phantom configurations, a PMMA plate $\left(15 \times 15 \mathrm{~cm}^{2}\right.$ area $)$ with thickness of $0.2 \mathrm{~cm}$ was located at the top of the phantom block containing the cavities. Additionally, four thick PMMA pieces were inserted around the phantom to press tightly together the structure and avoid possible air gaps between PMMA slabs or between the small pieces building each arranged cavity.

Two set-ups were used to perform the dosimetric measurements as illustrated in Fig. $1 \mathrm{a}$ and b. In one case, the $0.3 \mathrm{~cm}$ thickness slabs were positioned parallel to the beam axis in order to
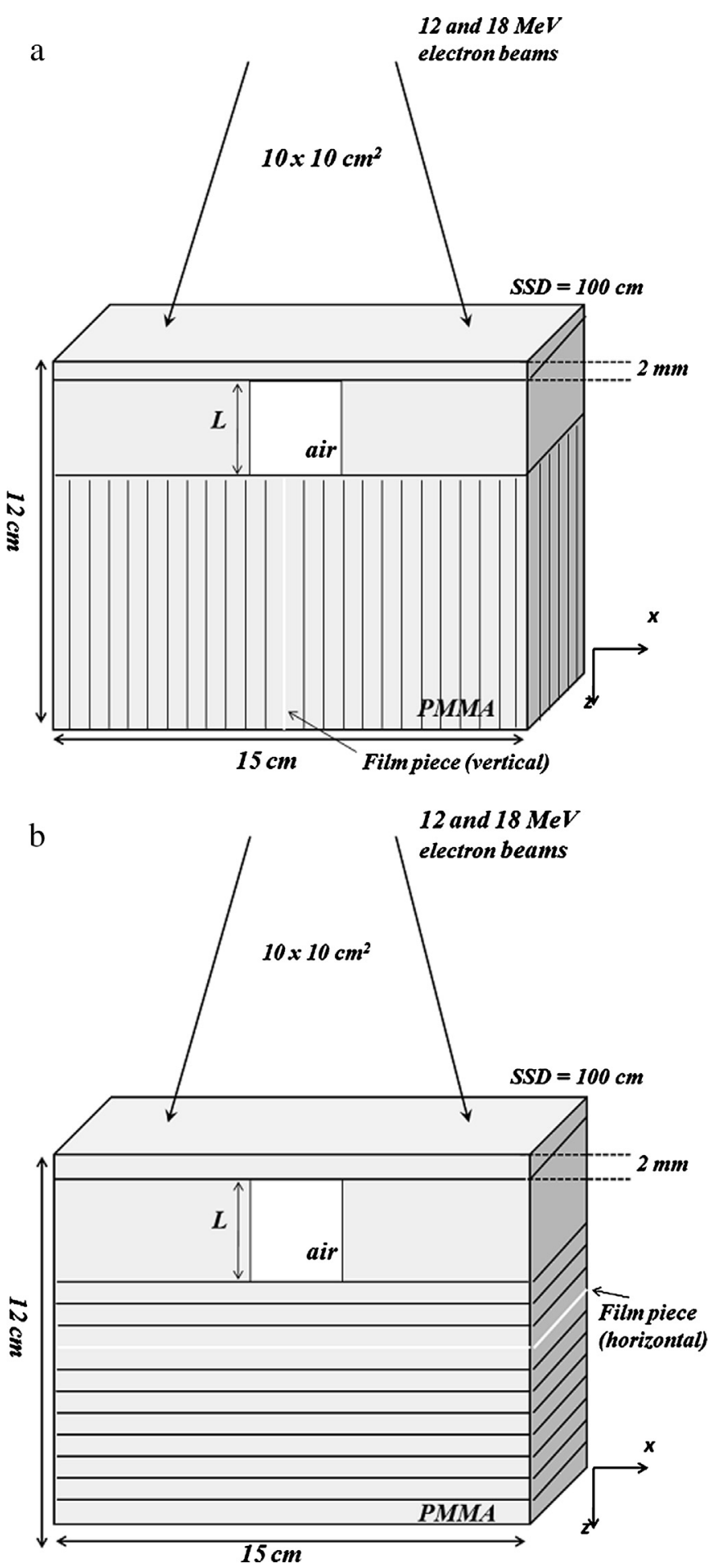

Figure 1. Scheme of sagittal (a-b) views of the home-built PMMA phantom including an air cavity with area $S$ and thickness $L$ used for experimental measurements and modeled for MC dose calculations (drawings not in scale). The cavities were located at $2 \mathrm{~mm}$ depth from the phantom surface. The white line indicates the position of the radiochromic film during experimental measurements. The electron beam was incident perpendicularly to the phantom surface, except for experimental acquisition of depth dose distribution (a) that a beam gantry angle of $2^{\circ}$ in respect to the film axis was to avoid the self-attenuation of the film.

acquire the depth dose curve (Fig. 1a). In the other case, the slabs were positioned perpendicularly to the beam for the acquisition of the transversal dose profiles (Fig. 1b). In both cases, the total thickness below the cavity was about $10 \mathrm{~cm}$ in order to consider 
Table 1

Thicknesses $(L)$ and areas $(S)$ of the cavities considered in the study and illustrated schematically in Fig. 1.

\begin{tabular}{ll}
\hline Square area, $S\left(\mathrm{~cm}^{2}\right)$ & Thickness, $L(\mathrm{~cm})$ \\
\hline $3.8 \times 3.8$ & 1.8 \\
$1.8 \times 1.8$ & 1.8 \\
$1 \times 1$ & 1.8 \\
$1 \times 1$ & 2.8 \\
$1 \times 1$ & 0.8 \\
\hline
\end{tabular}

the backscatter contribution. Further details of the experimental set-ups are described in Section 2.2.

\section{Radiochromic film measurements}

Radiochromic films type Gafchromic EBT2 were irradiated to determine the depth dose curves along the central axis and dose profiles at various depths for homogeneous and heterogeneous geometries. Measurements in the above described home-built PMMA phantom were carried out for electron beams of $12 \mathrm{MeV}$ and $18 \mathrm{MeV}$ from the Siemens Primus accelerator installed at the Virgen Macarena Hospital in Seville (Spain). A $10 \times 10 \mathrm{~cm}^{2}$ applicator size was applied at a source-to-surface distance (SSD) equal to $100 \mathrm{~cm}$.

For the depth dose curves acquisition in heterogeneous phantoms, pieces of radiochromic films $\left(12.5 \times 10 \mathrm{~cm}^{2}\right)$ were individually exposed parallel to the central axis of the incident beams (150 MU) between two central PMMA slabs of $0.3 \mathrm{~cm}$ thickness, which were vertically placed as illustrated in Fig. 1a. Depth dose curves were measured at depths below the PMMA block containing the cavity, i.e. beyond $3 \mathrm{~cm}$. A beam gantry angle of $2^{\circ}$ respect to the film axis was applied in order to avoid the self-attenuation of film and to reduce the effect of possible gaps between the phantom slabs $[17,18]$.

For measurements of depth dose curves in a homogeneous phantom, the PMMA block with the air cavity as well as the $2 \mathrm{~mm}$ thick plate were removed. Thus, a total depth dose distribution from the top surface of the phantom was registered in this case. Film samples were exposed following the same conditions described above for the heterogeneous phantom.

For transverse dose profile measurements, film samples $(12.5 \times$ $10 \mathrm{~cm}^{2}$ ) were irradiated horizontally on the central beam axis with $150 \mathrm{MU}$, supported between two PMMA slabs, at $3.3 \mathrm{~cm}$ and $4.2 \mathrm{~cm}$ depths.

Dose calibration curves of the film were obtained for both electron beam energies, 12 and $18 \mathrm{MeV}$, in order to evaluate the energy dependence of the film reported by previous studies [1922 ]. Overall, no significant differences (1.5\%) in film response for the entire dose range were observed between both energies. However, in order to minimize the energy dependence, the measurements were obtained by using the calibration curve for each corresponding energy.

An Epson 10000 XL Expression flatbed scanner was used to study the films response. After irradiation, the films were left for a period of $12 \mathrm{~h}$ before the scanning. During the scanning process, each film sample was placed on the center of the scan bed always with a portrait orientation and with the same side of the film facing the light source [22]. The images were scanned in transmission mode with a resolution of $75 \mathrm{dpi}(0.34 \mathrm{~mm} / \mathrm{pixel})$ in the 48 bit RGB uncompressed tagged image file format TIFF. Scanner warm-up effects were reduced by doing five successive pre-scans before the final reading of the film. The dosimetric analyses of scanned images were carried out using the Image [23] and UTOPIA [24] software. A median and mean filter $(3 \times 3)$ was applied to reduce noise. Moreover, the scanned images were converted into dose maps using a new method using multiple color channels, which compensates for a variety of anomalies, artifacts, and other disturbances, such as variations of the thickness of the active layer, scanner nonlinearity and process noise and enables the use of the entire available sensitivity range of the film in the same procedure [25].

In addition to the cares mentioned above, other reported guidelines and precautions about radiochromic dosimetry [26-28] were taken into account to ensure maximum reproducibility and accuracy of the results. All films used in this study were from the same batch to remove any variability between batches. They were always handled with gloves, and care was taken to avoid mechanical strain where possible. While not in use, films were stored in light-tight envelopes under constant atmospheric conditions. The EBT2 film uncertainty is considered to be about 3\% according to recent work [22].

\section{Monte Carlo simulations}

The EGSnrc Monte Carlo (MC) system code [29] was used for our MC calculations. The previous BEAMnrc/EGSnrc [30] simulated phase-space file [16] for a Siemens Primus linear accelerator installed at the Virgen Macarena Hospital in Seville was used as input of the DOSXYZnrc code [31] in order to calculate dose distributions in both homogeneous and heterogeneous phantom. These phase-space files correspond to the surface of the phantom (SSD of $100 \mathrm{~cm}$ ) for a $10 \times 10 \mathrm{~cm}^{2}$ electron beam of energies 12 and $18 \mathrm{MeV}$. Approximately 11 and 7 million of particles were collected in these files for the $12 \mathrm{MeV}$ and $18 \mathrm{MeV}$ electron beam. The uncertainty in the calculated electron fluence on the central axis $(0<r<4 \mathrm{~cm})$ is about $0.35 \%$ for the $12 \mathrm{MeV}$ beam and $0.3 \%$ in the18 MeV beam. Each dose calculation was run using a $2.2 \mathrm{GHz}$ AMD Opteron processor at the Nuclear Physics Centre of the University of Lisbon (Portugal).

To benchmark the matching degree of the accelerator simulations, the phase-space files were checked by comparing against experimental measurements, the phase-space files were indirectly checked by comparing against ionization chambers and film measurements. Overall, the MC calculated PDD and lateral profiles agree within $2 \%$ with ionization chamber measurements.

Monte Carlo phantoms were built using the same dimensions and materials as those phantoms described previously in the experimental setup (Table 1 ). The relative numbers of elemental compositions of PMMA, namely $\mathrm{H}=8 ; \mathrm{C}=5$ and $\mathrm{O}=2$, and density of $1.19 \mathrm{~g} / \mathrm{cm}^{3}$ were considered by the PEGS4 data-preprocessing code [29] to create the data files containing information of material cross section and branching ratios. The low energy thresholds for the production of knock-on electrons were set to $\mathrm{AE}=0.521 \mathrm{MeV}$ (total energy) and the threshold for bremsstrahlung events was set to $\mathrm{AP}=0.010 \mathrm{MeV}$.

In all EGSnrc calculations, the global electron energy cut-off (ECUT) and the global photon energy cut-off (PCUT) were set to $0.561 \mathrm{MeV}$ and $0.01 \mathrm{MeV}$, respectively and the default parameters for PRESTA were used, e.g. PRESTA-II for the electron-step algorithm and PRESTA-I for the boundary crossing algorithm. The low energy thresholds for the production of knock-on electrons and bremsstrahlung events were set to $\mathrm{AE}=0.521 \mathrm{MeV}$ (total energy) and $\mathrm{AP}=0.010 \mathrm{MeV}$. Auxiliary simulations considering an ECUT of $0.521 \mathrm{MeV}$ were also performed in order to analyze the impact of this parameter on the results. No significant dose differences were observed, whereas the simulation time was approximately halved.

The calculation dose-scoring voxel was set to $0.3 \mathrm{~cm}$ in $X, Y$ direction and $0.2 \mathrm{~cm}$ along $Z$ direction for the simulations performed in the homogeneous phantoms for both energies. For the heterogeneous phantoms, the same voxel size as used for the homogeneous phantom was set $\left(0.3 \times 0.3 \times 0.2 \mathrm{~cm}^{3}\right)$ in the lateral regions out the edges of the cavity. Inside and below the cavity (i.e. the acrylic region along the $Z$ axis limited by the cavity edges), the voxel size was set to 
$0.2 \times 0.2 \times 0.2 \mathrm{~cm}^{3}$ in order to reproduce accurately the details of the dose profiles in the immediate vicinity of the cavity. The choice of this voxel was based on previous findings reported by Mora et al. [32] and Cygler et al. [14].

A total of $4.4 \times 10^{7}$ and $3.4 \times 10^{7}$ histories were run for all the dose calculations using the phase space files for $12 \mathrm{MeV}$ and $18 \mathrm{MeV}$ beams, respectively. In each calculation, the phase space files were recycled only $3(12 \mathrm{MeV})$ and $4(18 \mathrm{MeV})$ times to avoid systematic bias [33].

The statistical uncertainty of calculated dose distributions was $1 \%(12 \mathrm{MeV})$ and $\sim 1.7 \%(18 \mathrm{MeV})$ at the maximum dose value for the homogeneous phantom. For the heterogeneous phantoms, the statistical uncertainty at the position of the maximum dose increased up to $1.7 \%(12 \mathrm{MeV})$ and $2.2 \%(18 \mathrm{MeV})$; whereas inside the cavity, the average uncertainty was about 3 and $4 \%$ for $12 \mathrm{MeV}$ and $18 \mathrm{MeV}$, respectively.

\section{Pencil beam dose calculations}

The Hogstrom pencil beam (PB) algorithm [34] implemented in the Philip Pinnacle (version 8.0) treatment planning system was used to calculate the dose distributions for the same phantom geometries as previously calculated with Monte Carlo simulations. Dose distributions were performed for electron beams of 12 and $18 \mathrm{MeV}$ and a $10 \times 10 \mathrm{~cm}^{2}$ field size. The phantoms were directly created in the TPS and the calculation voxel size was $0.2 \times 0.2 \times$ $0.2 \mathrm{~cm}^{3}$ for all phantoms.

\section{Results and discussion}

Dosimetric effect of an air cavity for $10 \times 10 \mathrm{~cm}^{2}$ electron beams

\section{Central-axis PDD variation}

Figures 2 and 3 compare the calculated central-axis PDD curve with the measured PDD curve for heterogeneous phantoms irradiated by electron beams of $12 \mathrm{MeV}$ and $18 \mathrm{MeV}$, respectively. The heterogeneous phantoms included an air cavity of varying size of thickness $L$ or area $S$, as presented in Table 1 . The beams were incident perpendicular to the phantom surface with a field size of $10 \times 10 \mathrm{~cm}^{2}$ at a SSD of $100 \mathrm{~cm}$. In addition, the PDD curve for the homogeneous phantom without air cavity (labeled as "without cavity") is also shown in these figures. All curves were normalized to the dose at the depth of dose maximum in the homogeneous phantom, i.e. $2.3 \mathrm{~cm}$ for $12 \mathrm{MeV}$ and $2.7 \mathrm{~cm}$ for $18 \mathrm{MeV}$. Note that the position of the cavities is kept at the same depth $(2 \mathrm{~mm}$ from phantom surface) while the dimensions $S$ or $L$ vary.

In Figs. 2 and 3, it can be clearly seen that the PDD is strongly dependent on the thickness $L$ of the cavity for both energies. As shown in Fig. 2a for $12 \mathrm{MeV}$ electron beams, the presence of an air cavity of $2.8 \mathrm{~cm}$ thickness leads to an enhancement of about $72 \%$ of the dose maximum with respect to the "without cavity" curve. Because of the small area of the cavity $\left(S=1 \times 1 \mathrm{~cm}^{2}\right)$, electrons scattered from the two PMMA sides surrounding the cavity can escape from the air cavity and contribute to the electron on-axis fluence at the first centimeters depths beyond the air-PMMA interface. As the cavity thickness decreases, the scatter contribution from the PMMA adjacent to the cavity decreases and hence the perturbation of the dose maximum is reduced along the central axis. For instance, the increase of dose maximum relative to the curve for the homogeneous phantom is only about $27 \%$ for the cavity phantom of $L=0.8 \mathrm{~cm}$ irradiated by a $12 \mathrm{MeV}$ beam.

It can be also seen from Fig. 2a that the presence of the air cavity with varying thickness causes a shift of the PDD curve to downstream direction. The reduced attenuation of electrons passing through the air compared to those through PMMA results in higher
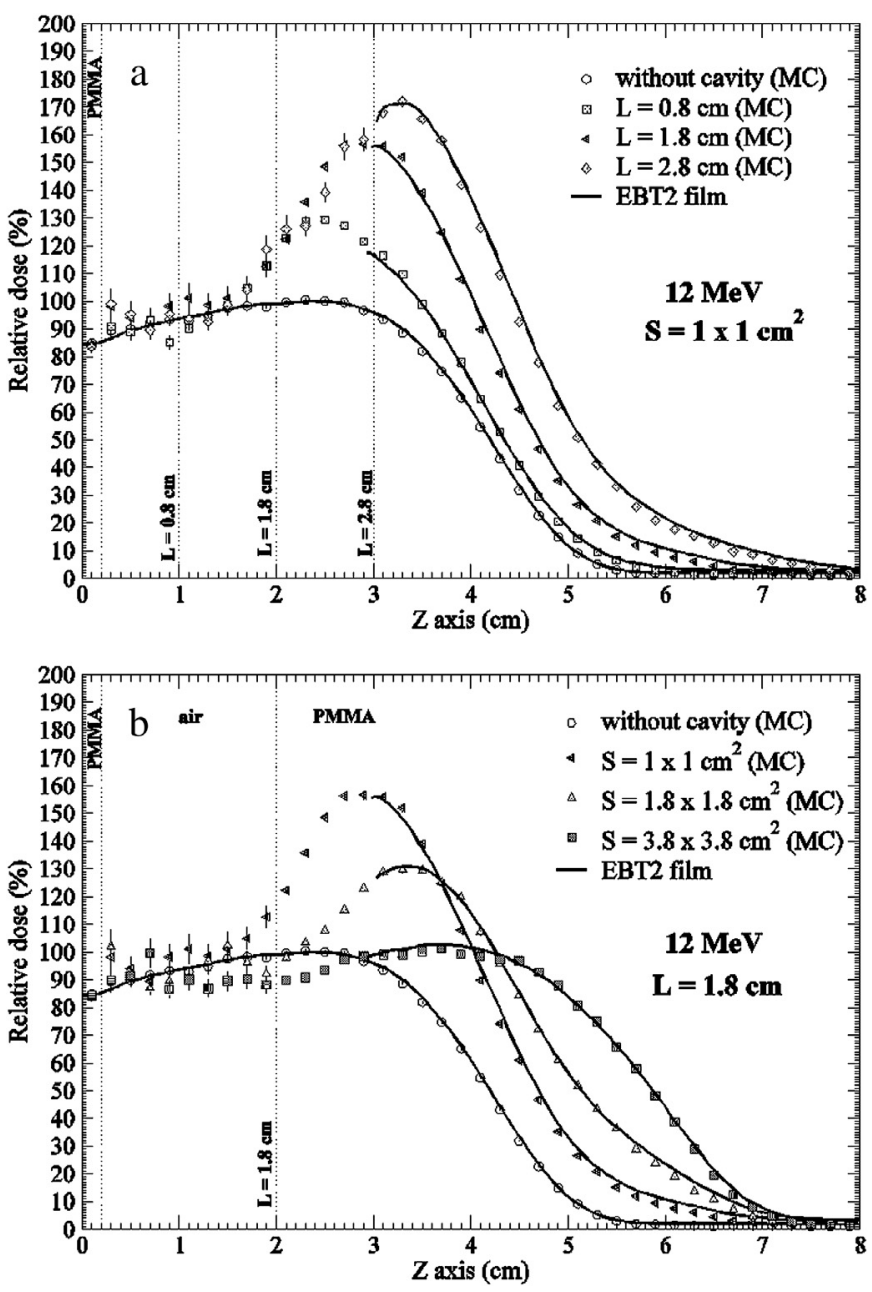

Figure 2. On-axis PDD curves measured (solid line) and MC calculated in heterogeneous phantoms including (a) a $1 \times 1 \mathrm{~cm}^{2}$ air cavity with different thickness $L$ and (b) a $1.8 \mathrm{~cm}$ thick air cavity with different area $S$. The top of the air cavities was located at $2 \mathrm{~mm}$ depth. An electron beam of $12 \mathrm{MeV}\left(10 \times 10 \mathrm{~cm}^{2}\right)$ was incident perpendicular to the phantom surface at a SSD of $100 \mathrm{~cm}$. The PDD curve for the homogeneous phantom (labeled as "without cavity") is also shown. Both MC calculated and experimental PDD curves are normalized to the maximum dose value of the PDD for the homogeneous phantom. The vertical lines show the position of the air cavities.

energy and forward electrons. This contribution of high energy electrons leads to the forward shift of the depth dose to deeper positions. As expected, the depth dose displacement becomes much pronounced for larger cavity thickness. As shown in Fig. 2a, the position of the dose maximum changes from $2.3 \mathrm{~cm}$ to $3.3 \mathrm{~cm}$ for the cavity of larger thickness $(L=2.8 \mathrm{~cm})$, whereas a small shift (2 mm) occurs for the cavity with thickness of $0.8 \mathrm{~cm}$.

Similar cavity effects on the PDD curves with the cavity thickness are also observed for the electron beam of $18 \mathrm{MeV}$, as illustrated in Fig. 3 for the cavity of $1 \times 1 \times 2.8 \mathrm{~cm}^{3}$. However, it is evident from this figure that the effect on the dose maximum is not so strong as those observed for the previous energy. In particular, the enhancement of the dose maximum due to the air cavity is reduced about $6 \%$ compared to $12 \mathrm{MeV}$ case for all heterogeneous phantoms. This reduction is probably due to the contribution of the electrons scattered from the PMMA sides surrounding the cavity to the on-axis electron fluence decreasing with the energy of the electron beam.

Figures $2 \mathrm{~b}$ and 3 show the dependence of the perturbation of depth dose on the variation of the square area $S$ of the cavity for 12 and $18 \mathrm{MeV}$ electron beams, respectively. As seen, the presence of 


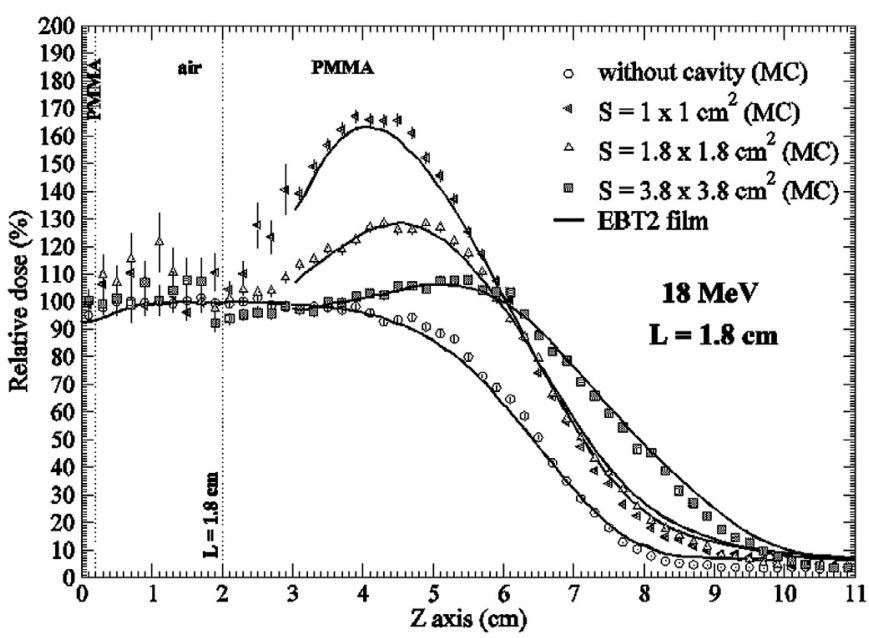

Figure 3. Central axis PDD curves measured (solid line) and MC calculated in heterogeneous phantoms including a $1 \times 1 \times 2.8 \mathrm{~cm}^{3}$ (left triangle), a $3.8 \times 3.8 \times 1.8 \mathrm{~cm}^{3}$ (square) and $1 \times 1 \times 1.8 \mathrm{~cm}^{3}$ (left triangle) air cavity. The top of the air cavities was located at $2 \mathrm{~mm}$ depth. An electron beam of $18 \mathrm{MeV}(10 \times 10 \mathrm{~cm} 2)$ was incident perpendicular to the phantom surface at a SSD of $100 \mathrm{~cm}$. The PDD curve of the homogeneous phantom (labeled as "without cavity") is also shown. Both MC calculated and experimental PDD curves are normalized to the maximum dose value of the PDD for the homogeneous phantom. The vertical lines show the position of the air cavities.

these air cavity configurations results in an increase of dose maximum as well as a shift forward of the PDD, analogously to the previous effect of cavities with varying thickness.

In particular, it is observed that the perturbation on the dose maximum value is reduced with the area of the cavity. For the $12 \mathrm{MeV}$ electron beam, the dose maximum increases about $57 \%$ for the cavity of $S=1 \times 1 \mathrm{~cm}^{2}$. This increase is smaller for higher values of $S$, attaining $32 \%$ for $S=1.8 \times 1.8 \mathrm{~cm}^{2}$ (Fig. 2 b) and about $1 \%$ for the wider cavity $\left(3.8 \times 3.8 \mathrm{~cm}^{2}\right)$. This is because the contribution at the central axis of the electrons scattered from the two PMMA lateral boundaries of the cavity is reduced significantly as the area increases.

On the other hand, it can be also noted that when the energy of the electron beam is increased to $18 \mathrm{MeV}$, the dose maximum perturbation for the cavities of $1 \times 1$ and $1.8 \times 1.8 \mathrm{~cm}^{2}$ is less pronounced (6-10\%) than that for the $12 \mathrm{MeV}$ beam. This behavior changes however for the largest cavity $\left(3.8 \times 3.8 \mathrm{~cm}^{2}\right)$ where the dose maximum leads to an increase of $6 \%$ of the maximum dose in comparison with the increase of $1 \%$ previously obtained for the beam energy of $12 \mathrm{MeV}$.

Comparing the dose curves for homogeneous and heterogeneous phantoms in Figs. 2b and 3, it is observed a shift of the PDD to deeper positions of the phantom caused by the presence of air cavities with varying area and this displacement of the PDD increases with the area of the cavity and the energy. So, for the $12 \mathrm{MeV}$ electron beam, a change of about $1.4 \mathrm{~cm}$ in the maximum dose position is observed when an air cavity of $3.8 \times 3.8 \mathrm{~cm}^{2}$ is embedded in the phantom with respect to that dose maximum position for the homogeneous phantom. Larger displacement (about $2.4 \mathrm{~cm}$ ) of dose maximum is found for $18 \mathrm{MeV}$ electron beam due to the longer range of the electrons for this energy.

The agreement between calculated and measured central-axis PDD is within $2 \%$ for all heterogeneous phantoms irradiated by a $12 \mathrm{MeV}$ electron beam as seen in Fig. 4. For $18 \mathrm{MeV}$, an agreement of $5 \%$ is found for all heterogeneous phantoms, except for the phantom with the $1 \times 1 \times 1.8 \mathrm{~cm}^{3}$ cavity where discrepancies between the measured and calculated values reach up to $7 \%$ in the region of maximum dose value (Fig. 3). These discrepancies may be caused by the inhomogeneity and non-uniform response of the film as reported by Hogstrom et al. [34].
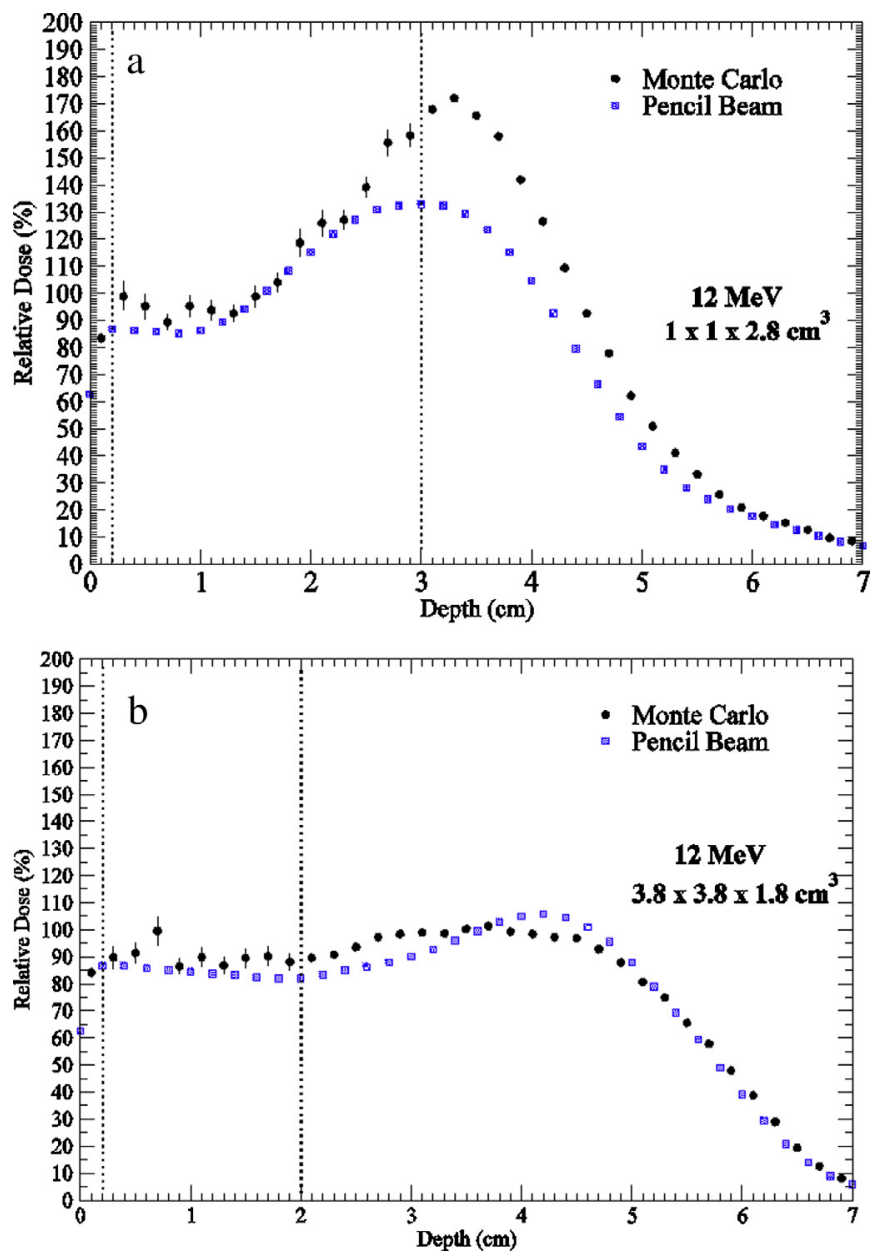

Figure 4. Central axis PDD curves calculated with the PB (blue square) and MC (black circle) algorithms in a heterogeneous phantom including a (a) $1 \times 1 \times 2.8 \mathrm{~cm}^{3}$ and (b) $3.8 \times 3.8 \times 1.8 \mathrm{~cm}^{3}$ air cavity. An electron beam of $12 \mathrm{MeV}\left(10 \times 10 \mathrm{~cm}^{2}\right)$ was incident perpendicular to the phantom surface at a SSD of $100 \mathrm{~cm}$. Both MC calculated and $\mathrm{PB}$ curves are normalized to the maximum dose value of the corresponding PDD for the homogeneous phantom. The vertical lines show the position of the air cavities (For interpretation of the references to colour in this figure legend, the reader is referred to the web version of this article.)

Figure 4 shows the comparison between central PDD curves calculated with MC and PB algorithms for $12 \mathrm{MeV}$ electron beam incident on a heterogeneous phantom with a $1 \times 1 \times 2.8 \mathrm{~cm}^{3}$ and $3.8 \times 3.8 \times 1.8 \mathrm{~cm}^{3}$ cavity. As seen, the PB algorithm underpredicts the maximum dose up to $40 \%$ with relation to the MC algorithms for the cavity of $1 \times 1 \times 2.8 \mathrm{~cm}^{3}$. Moreover, the dose displacement is reduced about $3 \mathrm{~mm}$ in the backward direction for the PDD curve calculated for the PB algorithm. These discrepancies between the $\mathrm{PB}$ and the MC become less significant as the thickness of the cavity decreases. For the cavity of $1 \times 1 \times 0.8 \mathrm{~cm}^{3}$, it was observed that the agreement between PB and MC PDD curves is within $2 \% / 2 \mathrm{~mm}$. These disagreements between both calculations may be attributed to the lack of electron scattering modeling of the PB algorithm, resulting therefore in a decrease of the scatter contribution to the central axis from the PMMA side adjacent to the cavity.

For the cavities with the largest area $\left(3.8 \times 3.8 \mathrm{~cm}^{2}\right)$, the PDD curve calculated with the PB algorithm shows discrepancies of about 5\% along the first centimeters after the cavity end in comparison with the MC-calculated PDD curve (Fig. 4b). In this case, it can be also observed that the maximum dose is shifted forward $3 \mathrm{~mm}$ with respect to MC calculations. These differences are reduced as the area of the cavity increases. 
The same behavior has been found for the electron beam of $18 \mathrm{MeV}$.

\section{Off-axis dose variation}

Figure $5 \mathrm{a}$ and $\mathrm{b}$ shows dose profiles calculated along the crossplane ( $X$ axis) of the PMMA heterogeneous phantoms (Table 1 ) and irradiated with electron beams of 12 and $18 \mathrm{MeV}$, respectively. The profiles are presented below the cavity at a depth of $3.3 \mathrm{~cm}$ for all cavity sizes. The figures include also the experimental profiles measured in the home-built PMMA phantom using EBT films at the same location as the calculated profiles. All curves were normalized to the central dose value of the homogeneous profile.

For $12 \mathrm{MeV}$, it is clearly seen that two small dips and peaks appear on the dose distribution near the lateral edge of the cavity with $3.8 \times 3.8 \mathrm{~cm}^{2}$ area and $1.8 \mathrm{~cm}$ thickness at the depth of $3.3 \mathrm{~cm}$ (Fig. 5a). This effect is caused by the existence of fewer electrons scattered from the air cavity into the surrounding PMMA than scattered into the air from the PMMA. For the other heterogeneous phantoms, it is observed a unique sharp peak along the central axis. For these cases, the decrease in cavity area leads to the increase in the fluence along the central axis of electrons scattered from the lateral cavity boundaries. The largest dose increase at the central
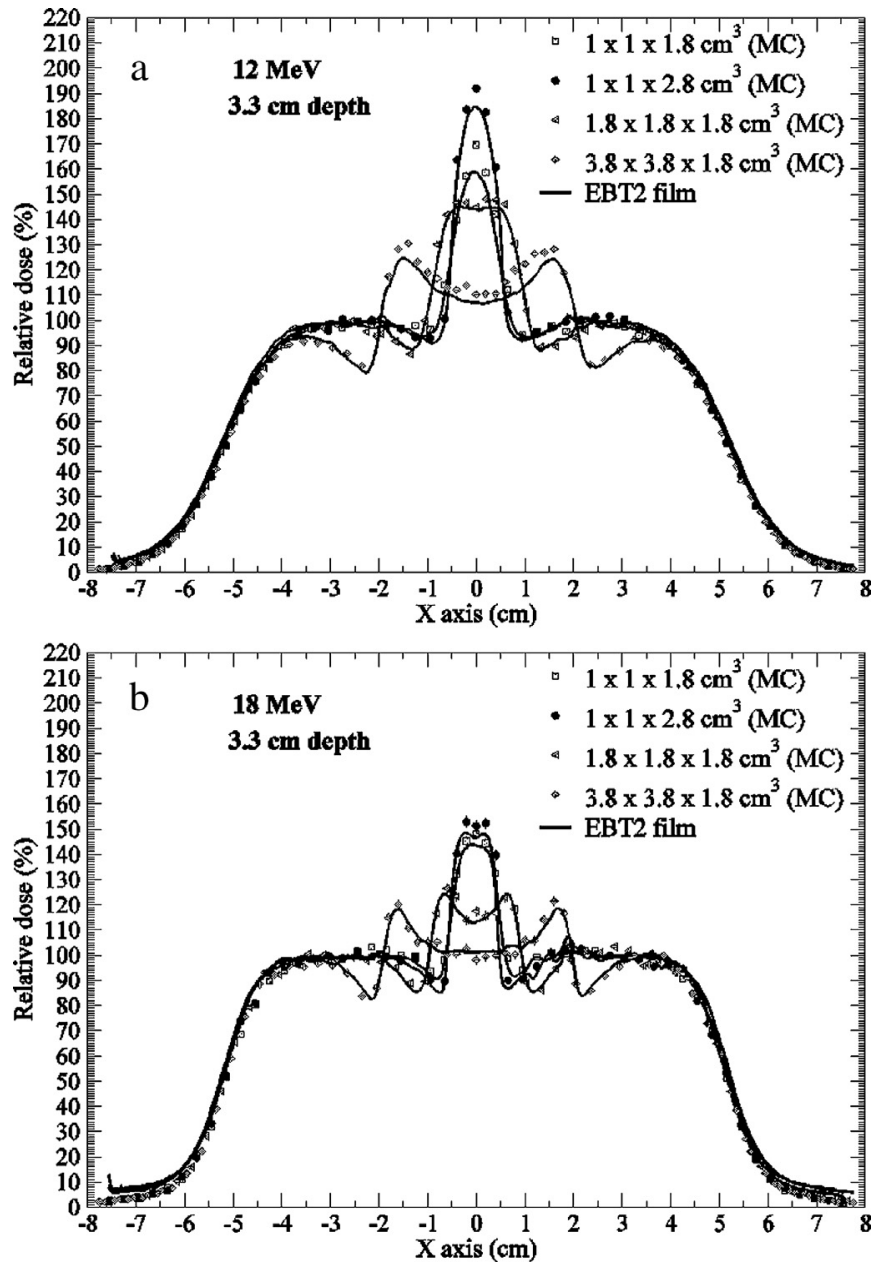

Figure 5. MC calculated (symbols) and measured (lines) cross-plane dose profiles ( $X$ axis) at $3.3 \mathrm{~cm}$ depth in heterogeneous phantoms containing an air cavity of different area $S$ and thickness $L$ and irradiated with an electron beam of $12 \mathrm{MeV}$ (a) and $18 \mathrm{MeV}$ (b). The beam was incident perpendicular to the phantom surface at a SSD of $100 \mathrm{~cm}$ and $10 \times 10 \mathrm{~cm}^{2}$ field size.

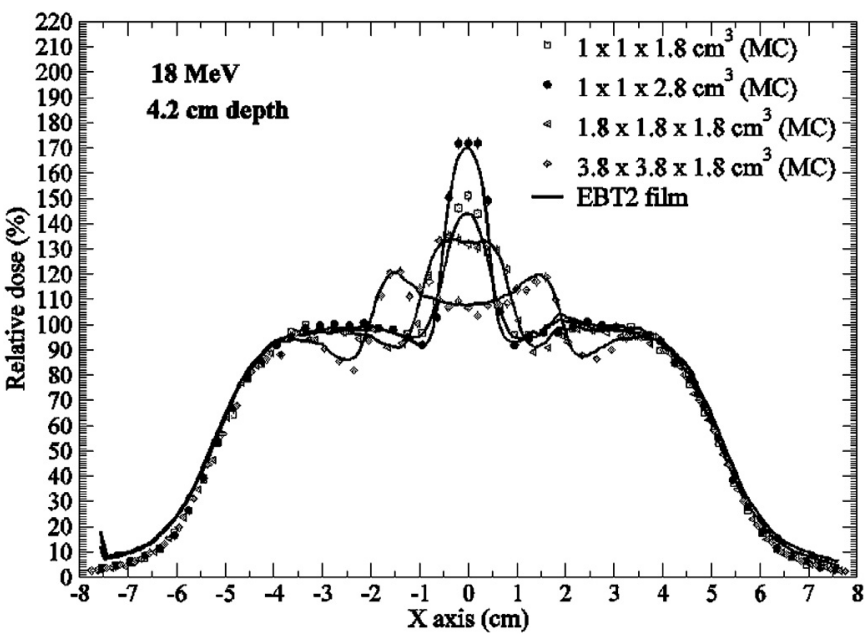

Figure 6. MC calculated (symbols) and measured (lines) cross-plane dose profiles ( $X$ axis) at $4.2 \mathrm{~cm}$ depth in heterogeneous phantoms containing an air cavity of different area $S$ and thickness $L$. An electron beam of $18 \mathrm{MeV}\left(10 \times 10 \mathrm{~cm}^{2}\right)$ was incident perpendicular to the phantom surface at a SSD of $100 \mathrm{~cm}$.
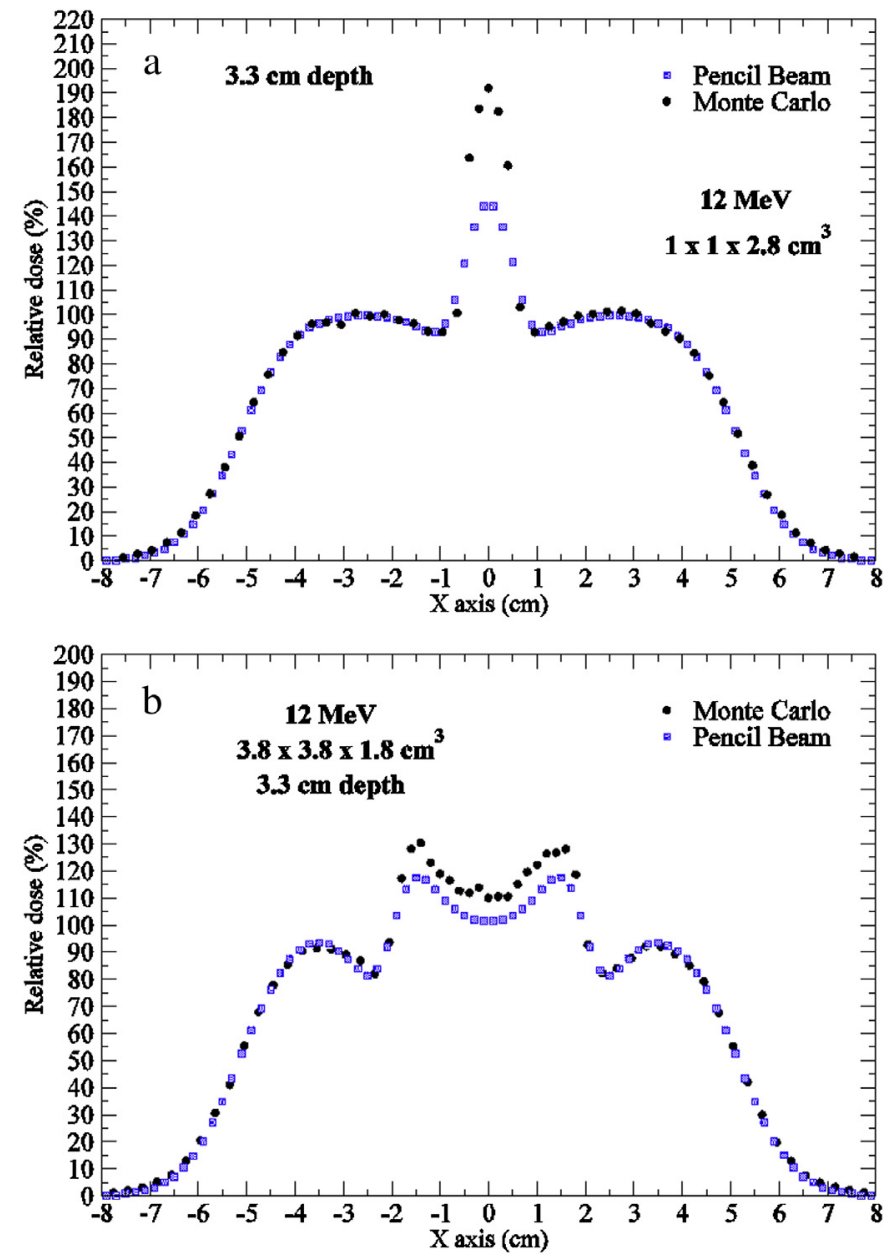

Figure 7. PB (blue square) and MC (black circles) calculated cross-plane dose profiles $(X$ axis) at $3.3 \mathrm{~cm}$ depth in heterogeneous phantoms containing an air cavity of (a) $1 \times 1 \times 2.8 \mathrm{~cm}^{3}$ and (b) $3.8 \times 3.8 \times 1.8 \mathrm{~cm}^{3}$. An electron beam of $12 \mathrm{MeV}\left(10 \times 10 \mathrm{~cm}^{2}\right)$ was incident perpendicular to the phantom surface at a SSD of $100 \mathrm{~cm}$. All curves were normalized to the central dose value of the homogeneous profile (For interpretation of the references to colour in this figure legend, the reader is referred to the web version of this article.) 
axis is caused by the presence of the largest thickness, $L=2.8 \mathrm{~cm}$, as exhibited previously by the PDD curves.

The effects of the electron disequilibrium caused by the air become less dramatic at greater distances below the cavity as shown in Fig. 6 for $18 \mathrm{MeV}$. At $4.2 \mathrm{~cm}$ depth, the characteristic dip and peak just around the cavity edges are significantly reduced and a sharp dose peak is clearly seen for all heterogeneous phantoms as a result of the increase of electron fluence at the center due the reduced attenuation of the electrons passing through the air cavity.

For $18 \mathrm{MeV}$, a similar behavior as previously mentioned for $12 \mathrm{MeV}$ is observed for the dose profiles at the depth of $3.3 \mathrm{~cm}$ in Fig. 5b.

From Figs. 5 and 6, it is also remarkable that experimental results show well the fine details of the dose near the interface of the cavity. For both energies, the agreement between measured and calculated is within $4-5 \%$ for all cavity sizes at both considered depths, except for the case of the $1 \times 1 \times 1.8 \mathrm{~cm}^{3}$ cavity at $12 \mathrm{MeV}$, where the difference between experimental and calculated profile at $3.3 \mathrm{~cm}$ depth reaches up to $10 \%$. Again, these discrepancies may be due to the nature of the measurement [34].

Figure 7 compares PB and MC calculated cross-plane dose profiles for the heterogeneous phantoms containing the cavity with the largest thickness $\left(1 \times 1 \times 2.8 \mathrm{~cm}^{3}\right)$ and the largest area $\left(3.8 \times 3.8 \times 1.8 \mathrm{~cm}^{3}\right)$ irradiated by a $12 \mathrm{MeV}$ electron beam. It is seen that the $\mathrm{PB}$ algorithm predicts the small dips and peaks of the dose distribution near the lateral edge, however, it underestimates the dose increase up to $40 \%$ for the cavity of $1 \times 1 \times 2.8 \mathrm{~cm}^{3}$ and $10 \%$ for the $3.8 \times 3.8 \times 1.8 \mathrm{~cm}^{3}$ cavity when compared to the MC algorithms. Similar results were found for the electron beam of $18 \mathrm{MeV}$.

\section{Conclusions}

Experimental measurements (Gafchromic EBT2 film), Pencil Beam and Monte Carlo simulations were used to evaluate systematically the dosimetric effect of a shallow air cavity embedded in a PMMA phantom as irradiated by electron beams of 12 and $18 \mathrm{MeV}$. The influence of this effect as a function of the cavity geometry and beam energy was specifically investigated using both methods for electron beams of 12 and $18 \mathrm{MeV}$ with the standard field size of $10 \times 10 \mathrm{~cm}^{2}$.

Significant dose increases up to $70 \%$ of the dose maximum were observed at depths below the cavities for both energies with respect to the homogeneous phantom. In addition to this perturbation, there was observed a shift on the depth-dose curve to larger depths which depends on the cavity geometry. Our results indicate that the variation and shift of the dose maximum caused by the presence of the air cavity on the depth dose curves were more pronounced for the low electron energy (12 MeV), large cavity thickness and small cavity area.

On the other hand, the shape of dose transversal profiles changes significatively due to the presence of an air cavity. In comparison to the homogeneous profile obtained for the phantom without air cavity, two small dips and peaks (about 10-20\%) for heterogeneous phantoms appear on the dose distributions near the lateral edge of the cavity.

The overall agreement between the MC calculations and EBT2 film measurements was about $4 \%$ for the depth-dose curves and offaxis profiles for the homogeneous and heterogeneous phantoms.

For both electron beam energies, the PB calculations have shown the same limitations to predict sharp high-dose variations for the heterogeneous phantom, in particular containing a cavity with a thickness of $2.8 \mathrm{~cm}\left(1 \times 1 \mathrm{~cm}^{2}\right.$ area $)$. Compared to MC results, the PB shows discrepancies of about $40 \%$ in the maximum dose for this cavity, decreasing with the cavity thickness. For the phantoms with wider cavities $\left(3.8 \times 3.8 \mathrm{~cm}^{2}\right)$, the PB reproduce well the increase of the maximum dose, however, it does not predict the displacement of the depth-dose curve.

In conclusion, MC calculations show much better agreements with Gafchromic EBT2 measurements and accurately predict the sharp dose increase behind the air cavities as well as the dose perturbation near the lateral edge of the cavities. However, it can be clearly seen how the PB calculations underpredict the dosimetric effects and their accuracy depends on the geometry of the air cavity.

The variations of the dose presented in this work due to the presence of shallow cavities can be therefore of clinical relevance. Treatment plans based on PB algorithm for cases of head and neck and others cases with shallow air inhomogeneities treated with electron beams should be carefully verified to avoid unaverted under or overdosage.

\section{Acknowledgments}

We would like to thank Dr. J. Cruz, A. Baiao and C. Mota for the support and help in design and construction of the PMMA phantom. This work was supported by Fundação para a Ciência e a Tecnologia grant.

\section{References}

[1] Al-Hallaq HA, Reft CS, Roeske JC. The dosimetric effects of tissue heterogeneities in intensity-modulated radiation therapy (IMRT) of the head and neck. Phys Med Biol 2006;51:1145-56.

[2] Wang L, Yorke E, Chui CS. Monte Carlo evaluation of tissue inhomogeneity effects in the treatment of the head and neck. Int J Radiat Oncol Biol Phys 2001;50:1339-49.

[3] Klein E, Lee MC, Rice RK, Mijnheer BJ. The influence of air cavities on interface doses for photon beams. Int J Radiat Oncol Biol Phys 1993;27: 419-26.

[4] NiroomandRad A, Harter KW, Thobejane S, Bertrand K. Air cavity effects on the radiation dose to the larynx using Co-60, $6 \mathrm{MV}$ and $10 \mathrm{MV}$ photon beams. Int J Radiat Oncol Biol Phys 1994;29:1139-46.

[5] Behrens CF. Dose build-up behind air cavities for Co-60, 4, 6 and 8 MV. Measurements and Monte Carlo simulations. Phys Med Biol 2006;51:5937-50.

[6] Li XA, Yu C, Homes T. A systematic evaluation of air cavity dose perturbation in megavoltage X-ray beams. Med Phys 2000;27:1011-7.

[7] Kan WK, Wu PM, Leung HT, Lo TC, Chung CW, Dora LWK, et-al. The effect of nasopharyngeal air cavity on X-ray interface doses. Phys Med Biol 1998;43: 529-37.

[8] Paelinck L, Reynaert N, Thierens H, De Wagter C, De Neve W. The value of radiochromic film dosimetry around air cavities: experimental results and Monte Carlo simulations. Phys Med Biol 2003;48:1895-905.

[9] Nüsslin F. The influence of air cavities on the dose distribution of high-energy electron beams. Phys Med Biol 1975;20:728-34.

[10] Shortt KR, Ross CK, Bielajew AF, Rogers DWO. Electron beam dose distributions near standard inhomogeneities. Phys Med Biol 1986;1:235-49.

[11] Zarza-Moreno M, Leal A, Teixeira N, Jesus AP, Mora G. Evaluation of air cavity effect for electron beams dose calculations using Monte Carlo method. Radiat Oncol 2007;84:S227.

[12] Chow JCL, Grigorov GN. Dosimetry of a small air cavity for clinical electron beams: a Monte Carlo study. Med Dosim 2010;35:92-100.

[13] Ding GX, Cygler JE, Christine WY, Kalach NI, Daskalov G. A comparison of electron beam dose calculation accuracy between treatment planning systems using either a pencil beam or a Monte Carlo algorithm. Int J Radiat Oncol Biol Phys 2005;63:622-33.

[14] Cygler JE, Daskalov GM, Chan G, Ding GX. Evaluation of the first commercial Monte Carlo dose calculation engine for electron beam treatment planning. Med Phys 2004;31:142-53.

[15] Ding GX, Duggan DM, Coffey CW, Shokrani P, Cygler JE. First macro Monte Carlo based commercial dose calculation module for electron beam treatment planning - new issues for clinical consideration. Phys Med Biol 2006;51: 2781-99.

[16] Salguero FJ, Arráns R, Palma BA, Leal A. Intensity- and energy-modulated electron radiotherapy by means of an xMLC for head and neck shallow tumors. Phys Med Biol 2010;55:1413-27.

[17] Khan FM, Doppke KP, Hogstrom KR, Kutcher GJ, Nath R, Prasad SC, et-al. Clinical electron beam dosimetry: report of AAPM radiation therapy committee task group No. 25. Med Phys 1990;18:73-109.

[18] Suchoweska N, Hoban P, Butson M, Davison A, Metcalfe P. Directional dependence in film dosimetry: radiographic and radiochromic film. Phys Med Biol 2001;46:1391-7. 
[19] Su Fan-Chi, Liu Y, Stathakis S, Shi C, Esquivel C, Papanikolaou N. Dosimetry characteristics of Gafchromic EBT film responding to therapeutic electron beam. Appl Radiat Isot 2007;65:1187-92.

[20] Sutherland JGH, Rogers DWO. Monte Carlo calculated absorbed - dose energy dependence of EBT and EBT2 film. Med Phys 2010;37:1110-6.

[21] Arjomandy B, Tailor R, Anand A, Sahoo N, Gillin M, Prado K, et-al. Energy dependence and dose response of Gafchromic EBT2 film over a wide range of photon, electron and proton beam energies. Med Phys 2010;37: 1942-7.

[22] Aland T, Kairn T, Kenny J. Evaluation of a Gafchromic EBT2 film dosimetry system for radiotherapy quality assurance. Australas Phys Eng Sci Med 2011;34:251-60.

[23] Abramoff MD, Magalhaes PJ, Ram SJ. Image processing with ImageJ. Biophoton Int 2004;11:36-42.

[24] Dorado Rodríguez MP, Macías Jaén J, Suero Rodrigo MA, Ortiz Lora A, Terrón León JA, Wals Zurita AJ. Valoración cuantitativa de tratamientos en radioterapia externa con la función o índice gamma. Presentación del software UTOPIA. Rev Fís Méd 2007;8:29-32.

[25] Micke A, Lewis DF, Yu X. Multichannel film dosimetry with nonuniformity correction. Med Phys 2011;38:2523-34.

[26] Niroomand-Rad A, Blackwell CR, Coursey BM, Gall KP, Galvin JM, McLaughlin WL, et-al. Radiochromic film dosimetry: recommendations of
AAPM radiation therapy committee task group 55. Med Phys 1998;25: 2093-114.

[27] Devic S. Radiochromic film dosimetry: past, present, and future. Phys Med Eur J Med Phys 2011;27:122-34.

[28] Butson MJ, Yu PKN, Cheung T, Metcalfe P. Radiochromic film for medical radiation dosimetry. Mater Sci Eng R 2003;41:61-120.

[29] Kawrakow I, Mainegra-Hing E, Rogers DWO, Tessier F, Walters BRB. The EGSnrc code system: Monte Carlo simulation of electron and photon transport. NRCC report PIRS-701; 2010.

[30] Rogers DWO, Walters B, Kawrakow I. BEAMnrc user's manual. NRCC report PIRS-0509(A)revK; 2009.

[31] Walters B, Kawrakow I, Rogers DWO. DOSXYZnrc user's manual. NRCC report PIRS-794revB; 2009.

[32] Mora G, Pawlicki T, Maio A, Ma CM. Effect of voxel size on Monte Carlo dose calculations for radiotherapy treatment planning advanced Monte Carlo for radiation physics, particle transport simulation and applications. Proceedings of the Monte Carlo 2000 conference, Lisbon, October 2000; 2000. p. 549-54.

[33] Walters B, Kawrakow I, Rogers DWO. History by history statistical estimators in the BEAM code system. Med Phys 2002:29:2745-52.

[34] Hogstrom KR, Mills MD, Almond PR. Electron beam dose calculations. Phys Med Biol 1981;26:445-59. 